\title{
EXISTENCE OF SOLUTIONS IN A POPULATION DYNAMICS PROBLEM*
}

\author{
BY \\ GASTÓN E. HERNÁNDEZ ${ }^{1}$ \\ Pontificia Universidad Catolica de Chile, Santiago
}

\begin{abstract}
In this paper we show the existence of a solution for the Gurtin-MacCamy model in population dynamics with age dependence and diffusion. We also discuss the behavior of this solution.
\end{abstract}

1. Introduction. We consider the population dynamics model with age dependence and diffusion proposed by Gurtin and MacCamy in a series of papers [1-8]. In this theory $\rho(a, t, x)$ represents the population distribution of age " $a$ " at time " $t$ "; $x$ is the space variable. There is a birth law

$$
\rho(0, t, x)=\int_{0}^{\infty} \beta_{0}(a, u(x, t)) \rho(a, t, x) d a,
$$

where $u(x, t)=\int_{0}^{\infty} \rho(a, t, x) d a$ is the space population density. The initial age-space distribution $\rho(a, 0, x)=\rho_{0}(a, x)$ is assumed to be nonnegative, and the balance law is:

$$
\rho_{a}(a, t, x)+\rho_{t}(a, t, x)+\mu(a, u(x, t)) \rho(a, t, x)=-q_{x}(a, t, x)
$$

where $q(a, t, x)=-k \rho(a, t, x) u_{x}(x, t)$ is the diffusion velocity that corresponds to the case in which species disperse to avoid crowding.

It is also assumed that the death modulus $\mu(a, u)$ is independent of $a$, and that the birth modulus is $\beta_{0}(a, u)=\beta(u) e^{-\alpha a}$ which models the case in which individuals are more fertile at younger ages. The case in which the birth modulus $\beta$ and the death modulus $\mu$ do not depend on the actual population $u$, have been treated by MacCamy [8] in the case of limited environment.

\footnotetext{
* Received November 19, 1984.

${ }^{1}$ Supported in part by Dirección de Investigación de la Universidad Católica de Chile through DIUC Grant and by Fondo Nacional de Desarrollo Cientifico y Tecnológico.
} 
We integrate the differential equation from 0 to $\infty$, with respect to $a$, assuming that $\rho(a, t, x)$ tends to 0 as $a$ tends to $\infty$. Also, we multiply it by $e^{-\alpha a}$ and integrate from 0 to $\infty$. We arrive at

$$
\begin{aligned}
u_{t}+\mu(u) u-\beta_{0}(u) G & =\left(u u_{x}\right)_{x}, \\
G_{t}+\left[\mu(u)+\alpha+\beta_{0}(u)\right] G & =\left(G u_{x}\right)_{x}, \\
u(x, 0) & =u_{0}(x), G(x, 0)=G_{0}(x),
\end{aligned}
$$

where $G$ is the auxiliary function

$$
G(x, t)=\int_{0}^{\infty} e^{-\alpha a} \rho(a, t, x) d a
$$

and

$$
u_{0}(x)=\int_{0}^{\infty} \rho_{0}(a, x) d a, \quad G_{0}(x)=\int_{0}^{\infty} e^{-\alpha a} \rho_{0}(a, x) d a .
$$

The natural assumption for $\beta$ and $\mu$ is that they are positive smooth functions, such that $\beta(u)$ is decreasing in $u$ and $\mu(u)$ is increasing in $u$. However, in this work we will assume only that both functions are uniformly bounded along with their first derivatives.

This problem is of most interest when the initial datum $u_{0}(x)$ has compact support, so we assume $u_{0}(x) \geqslant 0$ for $|x| \leqslant x_{1}$, and $u_{0}(x)=0$ for $|x| \geqslant x_{1}$. Also, from the definition of $u_{0}$ and $G_{0}$, we can see that if for some $x, \rho_{0}(a, x)=0$ almost everywhere in $a$, then $u_{0}(x)=G_{0}(x)=0$. Similarly if $\rho_{0}(a, x)>0$ in a set of finite measure, then $0<G_{0}(x)<$ $u_{0}(x)$.

We introduce the quotient function $q(x, t)$ defined by $q(x, t) u(x, t)=G(x, t)$ and $q_{0}(x, t) u_{0}(x, t)=G_{0}(x, t)$. With this new function the system becomes

$$
\left[\begin{array}{l}
\left(u u_{x}\right)_{x}+[\beta(u) q-\mu(u)] u=u_{t}, \\
q_{t}-u_{x} q_{x}=(\beta(u)-\alpha) q-\beta(u) q^{2}, \\
u(x, 0)=u_{0}(x), q(x, 0)=q_{0}(x),
\end{array}\right.
$$

with the assumptions $u_{0}(x) \geqslant 0$ and $0<\bar{m} \leqslant q_{0}(x) \leqslant 1$.

It is well known that even for the porous medium equation $\left(u u_{x}\right)_{x}=u_{t}$, with real analytic data, we can not expect to obtain classical solutions for all times, unless the initial data is strictly positive. We introduce a suitable definition of weak solutions for the system (1) and (2), in the following way:

Assume $u$ and $q$ are classical solutions of the system. Multiplying (1) by $q$, (2) by $u$, and substracting, we arrive at

$$
(u q)_{t}=\left[\frac{1}{2}\left(u^{2}\right)_{x} q\right]_{x}+(\beta(u)-\alpha-\mu(u)) u q .
$$

We let $\Omega_{T}=(-\infty, \infty) \times(0, T)$ and define a test function $\phi(x, t)$ as a continuously differentiable function in $\Omega_{T}$, with compact support in $\Omega_{T}$, and that equals 0 near $T$. 
Then multiplying this equation and Eq. (1) by $\phi$ and integrating over $\Omega_{T}$, we obtain

$$
\begin{aligned}
& \iint_{\Omega_{T}}\left[\frac{1}{2}\left(u^{2}\right)_{x} \phi_{x}-u \phi_{t}\right] d x d t \\
& =\iint_{\Omega_{T}}[\beta(u) q-\mu(u)] u \phi d x d t+\int_{\mathbf{R}} u_{0}(x) \phi(x, 0) d x, \\
& \iint_{\Omega_{T}}\left[\frac{1}{2}\left(u^{2}\right)_{x} q \phi_{x}-u q \phi_{t}\right] d x d t \\
& =\iint_{\Omega_{T}}[\beta(u)-\alpha-\mu(u)] u q \phi d x d \xi+\int_{\mathbf{R}} u_{0}(x) q_{0}(x) \pi(x, 0) d x
\end{aligned}
$$

Thus, we define a weak solution of problem (1) and (2) as a pair of functions $u, q$ such that: $u$ is continuous in $\Omega_{T}, u^{2}$ is differentiable with respect to $x$ in the sense of distributions, $q$ belongs to $L_{\text {loc }}^{2}\left(\Omega_{T}\right)$ and $u$ and $q$ satisfy (4) for any function $\phi$ continuously differentiable in $\bar{\Omega}_{T}$, with compact support in $\bar{\Omega}_{T}$ that equals 0 near $T$.

In this work we answer in the affirmative the question of existence, proposed by Gurtin in [1] as an open problem.

The main difficulty of the proof lies in the fact that (1) is not a uniformly parabolic equation because $u_{0}(x)=0$ for $|x|$ larger than $x_{1}$. Thus, first we obtain approximating solutions $u_{\varepsilon}$ and $q_{\varepsilon}$, with positive initial data $u_{0}(x)+\varepsilon$ and $q_{0}(x)$. Still, it is not possible to solve the system directly even with positive smooth initial data because of the presence of the function $q$ in (1) which, being a solution of a first-order differential equation, does not allow good a priori estimates for the function $u$. Thus, we consider another approximation problem by taking $q_{(n)}$, a "nice" version of $q$, instead of $q$. Once we obtain classical solutions to this last problem, we let $n$ tend to $\infty$ and then let $\varepsilon$ tend to 0 .

To solve the approximating problems, we integrate the equation for $q$ along the characteristics defined by $-u_{x}$, obtaining an explicit expression for the solution, together with some estimates in the supremum norm of the difference of two such solutions. Some estimates for the gradient of $q$ are also obtained. Then, we substitute this solution in the first equation and solve for $u$. To this scheme we apply the Schauder Fixed Point Theorem to obtain a solution for the system.

2. Some lemmas and notations. Through this paper differentiation is indicated by subscripts, $\Omega_{T}=\{(x, t) / x \in \mathbf{R}, 0<t<T\}$, and $\bar{\Omega}_{T}=\{(x, t) / x \in \mathbf{R}, 0 \leqslant x \leqslant T\}$. $C^{2,1}\left(\Omega_{T}\right)$ is the Banach Space consisting of functions $u(x, t)$ defined in $\Omega_{T}$ with continuous second derivatives in $x$, and continuous first derivative in $t . C^{\alpha}\left(\Omega_{T}\right)$ is the space of functions $u(x, t)$ defined in $\Omega$, for which the $\alpha$-norm,

$$
|u|_{\alpha}=\sup _{\Omega}|u|+\sup _{\Omega} \frac{|u(x, t)-(y, s)|}{|x-y|^{\alpha}+|t-s|^{\alpha / 2}}
$$


is bounded in $\Omega_{t} ; C^{2+\alpha}\left(\Omega_{T}\right)$ is the space of functions $u(x, t)$ for which the $2+\alpha$ norm $|u|_{2+\alpha}=\left|u_{x x}\right|_{\alpha}+\left|u_{x}\right|_{\alpha}+\left|u_{t}\right|_{\alpha}+|u|_{\alpha}$ is bounded in $\Omega_{T} . K(a, b, c, \ldots)$ are constants that depend on $a, b, c$, etc. The following results will be used in the proof.

Lemma 1. (The Hölder property). Let $u \in C^{2.1}\left(\Omega_{T}\right)$ be a solution of

$$
\begin{aligned}
u u_{x x}+\left(u_{x}\right)^{2} & =h(x, t, u)(u-\varepsilon)=u_{t}, \\
u(x, 0) & =u_{0}(x)+\varepsilon,
\end{aligned}
$$

such that $\varepsilon \leqslant u \leqslant M,|h| \leqslant M_{1}, u_{x}, u_{t}$ are bounded and $\left|u_{0}, u_{0}\right| \leqslant M_{0}$. Then the $\alpha$-norm of $u$ is bounded by a constant $K_{1}$ depending only on $\alpha, M, M_{0}, M_{1}$ and $T$, i.e.,

$$
\frac{|u(x, t)-u(y, s)|}{|x-y|^{\alpha}+|t-s|^{\alpha / 2}} \leqslant K_{1}, \quad \text { for any } x, y \in \mathbf{R}, \quad s, t \in[0, T] .
$$

The proof of this lemma is given in [9] and consists in considering the function

$$
g(x, y, t, s)=\frac{|u(x, t)-u(y, s)|^{2 / \alpha}}{|x-y|^{2}+K|t-s|^{\alpha / 2}}
$$

at a point of maximum.

LEMmA 2. ( Bounds for the gradient of $u$ ). Let $u \in C^{2,1}\left(\Omega_{T}\right.$ ) be a solution of

$$
\begin{aligned}
u u_{x x}+\left(u_{x}\right)^{2}+(\beta(u) q-\mu(u))(u-\varepsilon) & =u_{t}, \\
u(x, 0) & =u_{0}(x)+\varepsilon .
\end{aligned}
$$

Assume that $\left|q_{x}\right| \leqslant N_{1},|q| \leqslant 1,|h(x, t, u)|=|\beta(u) q-\mu(u)| \leqslant M_{1}, \varepsilon \leqslant u \leqslant M$, and $u_{x}$, $u_{x x}$ bounded in $\Omega_{T}$. Then there exist constants $K_{2}$ and $K_{3}$ depending only on $M, M_{0}$, and $M_{1}$ such that for any $c$ positive, $\left|u_{x}\right|^{2} \leqslant K_{2}+K_{3} / c+c N_{1}^{2}$ in $\Omega_{T}$.

The proof of this lemma, also given in [9], is a direct application of Bershtein's technique to Eq. (6).

3. The $\varepsilon-n$-approximation problems. To prove existence of solutions of (1) and (2) we solve first the $\varepsilon$ - $n$-approximation problems;

$$
\begin{aligned}
& u u_{x x}+u_{x}^{2}+\left(\beta(u) q_{(n)}-\mu(u)\right)(u-\varepsilon)=u_{t}, \\
& q_{t}-u_{x} q_{x}=(\beta(u)-\alpha) q-\beta(u) q^{2}, \\
& u(x, 0)=u_{0}(x)+\varepsilon, \quad q(x, 0)=q_{0}(x),
\end{aligned}
$$

where $q_{(n)}$ is a "smoothed" version of $q$. That is to say, if $k$ is a nonnegative $C^{\infty}$ function in $\mathbf{R}^{2}$ such that $k(r)=0$ for $|r| \geqslant 1, k$ is symmetric in $r$ and $\iint k(r) d r=1$, we define $k_{n}(r)=n k(n r)$ and $q_{(n)}(r)=\iint k_{n}(r-s) q(r) d s$. We note that if $q \in L^{2}, q_{(n)}$ is a $C^{\infty}$ sequence of functions that converge to $q$ in $L^{2}$. If $q$ is continuous, $q_{(n)}$ converge to $q$ uniformly on compact sets.

To prove that this system has a solution we first study equation (8) in terms of existence, bounds for the gradient of $q$ and bounds for the difference of two solutions. Then we apply the Leray-Schauder Fixed Point Theorem as presented in Friedman [10] to obtain a classical solution of (7) and (8). 
The following two lemmas establish existence of solutions for Eq. (8) given the function $u$.

Lemma 3. Let $v \in C^{2+\alpha}\left(\Omega_{T}\right), \bar{m} \leqslant q_{0}(x) \leqslant 1$ and $|v|_{2+\alpha} \leqslant K_{4}$. Then the problem

$$
\begin{aligned}
q_{t}-v_{x} q_{x} & =(\beta(v)-\alpha) q-\beta(v) q^{2} \\
q(x, 0) & =q_{0}(x)
\end{aligned}
$$

has a unique solution $q(\bar{x}, \bar{t})$ in $\Omega_{T}$, given by

$$
q(\bar{x}, \bar{t})=e^{\int_{0}^{t}(\beta(v)-\alpha) d s}\left[\int_{0}^{i} \beta(v) e^{-\int_{0}^{t}(\beta(v)-\alpha) d s} d t+q_{0}^{-1}(X(0))\right]
$$

where $\beta(v)=\beta(v(x(t), t))$. The integration is along the characteristic curves defined by

$$
\frac{\partial X}{\partial t}=-v_{X}(x, t), \quad X(\bar{t} ; \bar{t}, \bar{x})=\bar{x} .
$$

Proof. We define characteristic functions $X(t ; \bar{t}, \bar{x})$ by means of (11). Since $v_{x}$ is Lipschitz continuous there is always a unique local solution of this equation. Further, since $v_{x}$ is also bounded in $\Omega_{T}$, this local solution can be made global by extending it to the boundary of $[0, T] \times \mathbf{R}$. The usual techniques of maximum principles can be applied to show that if $q, q_{x}$ are bounded in $\Omega_{T}$ and $\bar{m} \leqslant q_{0}(x) \leqslant 1$, then $\bar{m} e^{-\alpha t} \leqslant q(x, t) \leqslant 1$. In particular we expect $q$ to be a positive function. Let

$$
p(x, t)=q^{-1}(x, t) e^{\int_{0}^{t}[\beta(v(X(s), s))-\alpha] d s} .
$$

If we assume that $q$ is a solution of (9), then $p$ satisfies

$$
p_{t}-v_{x} p_{x}=\beta(x, t), \quad p(x, 0)=q_{0}^{-1}(x),
$$

where $\beta(x, t)=\beta(v(x, t)) e^{\int_{0}^{t}[\beta(v(X(t), s))-\alpha] d s}$.

Integrating this equation along characteristics from 0 to $\bar{t}$ we obtain

$$
p(\bar{x}, \bar{t})=p(X(0), 0)+\int_{0}^{t} \beta(X(s), s) d s .
$$

This, together with (12), gives expression (10) for $q(\bar{x}, \bar{t})$. The solution thus obtained is just a formal one, but it can be proved by direct differentiation and by the fact that

$$
X_{i}(t)-v_{x}(\bar{x}, \bar{t}) X_{\bar{x}}(t)=0 \text { for every } t,
$$

that (10) is a solution of (9). The uniqueness follows from Haar's lemma.

4. Estimates for $|p-q|$ and $\left|q_{x}\right|$.

Lemma 4. (Estimates for $|p-q|)$. Let $v, w \in C^{2+\alpha}(\Omega),|v, w|_{2+\alpha} \leqslant K_{4},|v-w|_{2+\alpha} \leqslant \delta$ and $p, q$ solutions of (9) with $v$ and $w$, respectively. That is,

$$
\begin{gathered}
q_{t}-v_{x} q_{x}=(\beta(v)-\alpha) q-\beta(v) q^{2}, \\
p_{t}-w_{x} p_{x}=(\beta(w)-\alpha) p-\beta(w) p^{2}
\end{gathered}
$$

with $q(x, 0)=p(x, 0)=q_{0}(x)$.

Then $|p-q| \leqslant K \delta$ where $K_{5}$ depends only on $K_{4}, T$, and $q_{0}$. 
Proof. Let $X_{1}(t ; \bar{t}, \bar{x})$ and $X_{2}(t ; \bar{t}, \bar{x})$ be the characteristics defined by

$$
\begin{array}{ll}
\frac{\partial X_{1}}{\partial t}=-v_{X}\left(x_{1}, t\right), & X_{1}(\bar{t})=\bar{x}, \\
\frac{\partial X_{2}}{\partial t}=-w_{X}\left(X_{2}, t\right), & X_{2}(\bar{t})=\bar{x} .
\end{array}
$$

Let $X_{3}(t)=\left|X_{1}(t)-X_{2}(t)\right|$. Then subtracting both equations in (18) and using Gronwall's inequality we obtain

$$
X_{3}(t) \leqslant T\left|v_{x}-w_{x}\right|_{\infty} e^{\left|r^{\prime},\right|_{x} T} \leqslant T e^{K_{4} T} \delta .
$$

Now, since $p$ and $q$ are given by the same expression (10) (with $\beta(v)$ for $q, \beta(w)$ for $p$, etc.), subtracting these formulae we arrive at an expression that contains multiples (depending on $K_{4},|\beta|_{\infty}, T$, etc.) of $\beta(v)-\beta(w)$. This in turn is bounded by $\beta^{\prime}, v-w$, and $X_{1}-X_{2}$, and is therefore bounded by a constant $K$ times $\delta$.

Lemma 5. (Bounds for the gradient of $q$ ). Let $u$ be as in Lemma 1 and $q$ given by (10), with $\partial X / \partial t=u_{X}(X, t)$ and $X(\bar{t})=\bar{x}$. Then $\left|q_{\bar{x}}\right| \leqslant K_{5}\left|u_{x}\right|_{\infty}$ and $\left|q_{\bar{t}}\right| \leqslant K_{5}\left|u_{x}\right|_{\infty}^{2}$, where $K_{5}=$ $K_{5}\left(\varepsilon, T, M_{1}, M_{0}\right)$.

Proof. Differentiating the characteristic equations with respect to the parameter $\bar{x}$, we obtain

$$
\partial X_{\bar{x}} / \partial t=-u_{X X}(X, t) X_{\bar{x}}, \quad X_{\bar{x}}(\bar{t})=1,
$$

so $X_{\bar{x}}=e^{\int_{1}^{\prime} u_{X X}(S(s) . s) d s}$. Using the differential equation for $u_{X X}$ and integrating, we obtain $\left|X_{\bar{x}}\right| \leqslant(M / \varepsilon) e^{T\left(M_{1}+1\right)}$. Then by $(15),\left|X_{i}\right| \leqslant(M / \varepsilon) e^{T\left(M_{1}+1\right)}\left|u_{x}\right|_{\infty}$. Differentiating the expression for $q(\bar{x}, \bar{t})$, we obtain that $q_{\bar{x}}$ is bounded by multiples of $\beta^{\prime}, u_{x}, X_{\bar{x}}, q_{0}^{-1}$, and $q_{0}^{\prime}$, so that $q_{\bar{x}}$ is bounded by a constant $K_{5}\left(\varepsilon, T, M_{1}, M_{0}\right)$ times $\left|u_{x}\right|_{\infty}$. The same argument is valid for $\left|q_{i}\right|$.

\section{Existence of solutions of the $\varepsilon$ - $n$-approximating problems.}

THEOREM 1. Under the given hypotheses for $u_{0}, q_{0}, \beta$, and $\mu$, there exists a classical solution in $\Omega_{T}$ of

$$
\begin{aligned}
u u_{x x}+u_{x}^{2}+\left(\beta(u) q_{(n)}-\mu(u)\right)(u-\varepsilon) & =u_{t}, \\
q_{t}-u_{x} q_{x} & =(\beta(u)-\alpha) q-\beta(u) q^{2}, \\
u(x, 0) & =u_{0}(x)+\varepsilon, q(x, 0)=q_{0}(x) .
\end{aligned}
$$

Proof. Let $V$ be the convex set in $C^{2+\alpha}\left(\Omega_{T}\right)$ consisting of all functions $w$ with $|w|_{2+\alpha} \leqslant K_{6}$ and $w \geqslant \varepsilon$. We define $T: V \rightarrow C^{2+\alpha}\left(\Omega_{T}\right)$ in the following way. Given $w \in W,|w|_{2+\alpha} \leqslant K_{4}$, by Lemma 3 there exists a unique solution $q$ of

$$
\begin{aligned}
q_{t}-w_{x} q_{x} & =(\beta(w)-x) q-\beta(w) q^{2}, \\
q(x, 0) & =q_{0}(x) .
\end{aligned}
$$

By the maximum principle, $m e^{-\alpha t} \leqslant q(x, t) \leqslant 1$. Thus $q_{(n)} \in C^{\infty}\left(\Omega_{T}\right)$ and $\left|q_{(n)}\right|_{\sigma} \leqslant 2 n$ for any $\sigma \in(0,1)$. Now consider the problem

$$
\begin{aligned}
E(u) u_{x x}+u_{x}^{2}+\left(\beta(u) q_{(n)}-\mu(u)\right)(u-\varepsilon) & =u_{t}, \\
u(x, 0) & =u_{0}(x)+\varepsilon,
\end{aligned}
$$


where $E(u)$ is a $C^{\infty}$ function of $u$ that equals $\frac{\varepsilon}{2}$ for $u \leqslant \frac{\varepsilon}{2}$; it increases up to $\varepsilon$ in $\left(\frac{\varepsilon}{2}, \varepsilon\right)$ and equals $u$ for $u \geqslant \varepsilon$. This problem has a unique solution $u \in C^{2+\sigma}\left(\Omega_{T}\right),|u|_{2+\sigma} \leqslant K(\varepsilon, n)$. By the maximum principle $\varepsilon \leqslant u \leqslant M(\varepsilon, n)$; then $E(u)=u$. Actually $u$ is the unique solution in $C^{2+\sigma}\left(\Omega_{T}\right)$ of Eq. (20) that satisfies these conditions. We let $u=T(w)$ and choose $K_{4} \geqslant K(\varepsilon, n)$ and $\sigma>\alpha$. Then $T$ maps $V$ into $V$. Since bounded sets in $C^{2+\sigma}\left(\Omega_{T}\right)$ are precompact in $C^{2+\alpha}\left(\Omega_{T}\right)$ with $0<\alpha<\sigma<1$, we obtain that $T(V)$ is precompact.

Next we prove that $T$ is continuous. We let $u=T(w) z=T(v)$. If we subtract the corresponding $q$ and $p$ we find that $|q-p|_{\infty} \leqslant K\left(K_{4}\right) \delta$ when $|v-w|_{2+\alpha} \leqslant \delta$. Thus $\left|q_{(n)}-p_{(n)}\right|_{\alpha} \leqslant K\left(K_{4}, n\right) \delta$. We then set $S=u-z$ and note that $S$ satisfies the linear equation

$$
\begin{aligned}
u S_{x x}+\left(u_{x}+z_{x}\right) S_{x}+c(x, t) S-S_{t} & =f(x, t), \\
S(x, 0) & =0,
\end{aligned}
$$

where

$$
c(x, t)=\left(\beta(u) q_{(n)}-\mu(u)\right)+(z-\varepsilon)\left(p_{(n)} \beta^{\prime}(\cdot)-\mu^{\prime}(\cdot)\right)+z_{x x}
$$

and

$$
f(x, t)=\beta(u)(z-\varepsilon)\left(q_{(n)}-p_{(n)}\right) .
$$

We also have that $u \geqslant \varepsilon, u_{x}+z_{x}$ is bounded by $K_{4}, c(x, t)$ is bounded by a multiple of $K_{4}$ and the $\alpha$-norm of $f(x, t)$ is bounded by a multiple of the $\alpha$-norm of $q_{(n)}-p_{(n)}$, i.e., it is bounded by $K \delta$. Then by Theorem 5.1 in [11] we have $|S|_{2+\alpha} \leqslant K\left(K_{4}, n, \varepsilon\right) \delta$. This implies continuity.

Thus $T$ has a fixed point in $V$, i.e., there exist functions $u$ and $q$ solutions of (20). Since $0<\bar{m} e^{-\alpha T} \leqslant q \leqslant 1$, by the maximum principle we obtain that $\varepsilon \leqslant u \leqslant M_{1}$, where $M_{1}=$ $\left(M_{0}+1\right) e^{M_{3} T}$ and $M_{3}=|\beta|_{\infty}+|\mu|_{\infty} .\left(M_{1}\right.$ is independent of $\varepsilon$ and $n$.)

Also

$$
\begin{array}{rlrl}
u \in C^{2+\alpha}\left(\Omega_{T}\right), & |u|_{2+\alpha} & \leqslant K(n, \varepsilon), \\
q \in C^{1+\alpha}\left(\Omega_{T}\right), & |q|_{1+\alpha} & \leqslant K(n, \varepsilon), \\
& |q|_{x},\left|q_{t}\right| \leqslant K\left(\left|u_{x}\right|_{\infty}, \varepsilon\right) .
\end{array}
$$

Existence of solutions of the $\varepsilon$-approximating problems. In this section we prove existence of solutions of the system:

$$
\begin{gathered}
\left(u u_{x}\right)_{x}+(\beta(u) q-\mu(u))(u-\varepsilon)=u_{t}, \\
q_{t}-u_{x} q_{x}=(\beta(u)-\alpha) q-\beta(u) q^{2}, \\
u(x, 0)=u_{0}(x)+\varepsilon, \quad q(x, 0)=q_{0}(x) .
\end{gathered}
$$

THEOREM 2. Under the given hypothesis on $\beta, \mu, u_{0}$, and $q_{0}$, there exists a (weak) solution of (21) and (22) for each $\varepsilon>0$. That is to say, there exists a continuous function $u$, $\left(u^{2}\right)_{x} \in L_{\mathrm{loc}}^{2}\left(\Omega_{T}\right)$ and a function $q, q \in L_{\mathrm{loc}}^{2}\left(\Omega_{T}\right)$, that satisfy

$$
\begin{aligned}
\iint_{\Omega_{T}}\left[\frac{1}{2}\left(u^{2}\right)_{x} \phi_{x}-\right. & \left.u \phi_{t}\right] d x d t \\
& =\iint_{\Omega_{T}}(\beta(u) q-\mu(u))(u-\varepsilon) d x d t+\int_{\mathbf{R}}\left(u_{0}(x)+\varepsilon\right) \phi(x, 0) d x
\end{aligned}
$$


and

$$
\begin{aligned}
\iint_{\Omega_{T}}\left[\frac{1}{2}\left(u^{2}\right)_{x} q \phi_{x}-u q \phi_{t}\right] d x d t=\iint_{\Omega_{T}} & {[(\beta(u)-\alpha-\mu(u)) u q \phi] d x d t } \\
& +\int_{\mathbf{R}}\left(u_{0}(x)+\varepsilon\right) q_{0}(x) \phi(x, 0) d x
\end{aligned}
$$

for every test function $\phi$ over $\Omega_{T}$.

Proof. By the previous theorem, for each $n$, we have $u^{n} \in C^{2+\alpha}\left(\Omega_{T}\right), q^{n} \in C^{1}\left(\Omega_{T}\right)$ such that

$$
\begin{gathered}
\left(u^{n} u_{x}^{n}\right)_{x}+\left[\beta^{n} q_{(n)}^{n}-\mu^{n}\right]\left(u^{n}-\varepsilon\right)=u_{t}^{n}, \\
q_{t}^{n}-u_{x}^{n} q_{x}^{n}=\left(\beta^{n}-\alpha\right) q^{n}-\beta\left(q^{n}\right)^{2}, \\
u^{n}(x, 0)=u_{0}(x)+\varepsilon, \quad q^{n}(x, 0)=q_{0}(x) .
\end{gathered}
$$

Multiplying (21) by $q^{n},(22)$ by $u^{n}$, and adding, we obtain

$$
\begin{aligned}
\left(u^{n} q^{n}\right)_{t}= & \frac{1}{2}\left[\left(\left(u^{n}\right)^{2}\right)_{x} q^{n}\right]_{x}+\left(\beta^{n}-\alpha-\mu^{n}\right) u^{n} q^{n} \\
& +\beta^{n} u^{n} q^{n}\left(q_{(n)}^{n}-q^{n}\right)-\varepsilon q^{n}\left(\beta^{n} q_{(n)}^{n}-\mu^{n}\right) .
\end{aligned}
$$

If $\phi$ is a test function we multiply (25) and (26) by $\phi$ and integrate over $\Omega_{T}$ to obtain

$$
\begin{aligned}
\iint_{\Omega_{T}}\left[\frac{1}{2}\left(\left(u^{n}\right)^{2}\right)_{x} \phi_{x}-u^{n} \phi_{t}\right] d x d t= & \iint_{\Omega_{T}}\left(\beta^{n} q_{(n)}^{n}-\mu^{n}\right)\left(u^{n}-\varepsilon\right) d x d t \\
& +\int_{\mathbf{R}}\left(u_{0}(x)+\varepsilon\right) \phi(x, 0) d x
\end{aligned}
$$

and

$$
\begin{aligned}
& \iint_{\Omega}\left[\frac{1}{2}\left(\left(u^{n}\right)^{2}\right)_{x} q^{n} \phi_{x}-u^{n} q^{n} \phi_{t}\right] d x d t \\
&=\iint_{\Omega_{T}}\left(\beta^{n}-\alpha-\mu^{n}\right) u^{n} q^{n} \phi d x d t+\iint_{\Omega_{T}}\left[\beta^{n} u^{n} q^{n}\left(q_{(n)}^{n}-q^{n}\right) \phi\right] d x d t \\
& \quad-\varepsilon \iint_{\Omega_{T}}\left[q^{n}\left(\beta^{n} q_{(n)}^{n}-\mu^{n}\right) \phi\right] d x d t+\int_{\mathbf{R}}\left(u_{0}(x)+\varepsilon\right) q_{0}(x) \phi(x, 0) d x .
\end{aligned}
$$

Since $\left|u^{n}\right|_{\alpha} \leqslant K_{1}$ uniformly in $n$, there exists a subsequence $\left\{u^{n_{1}}\right\}$ such that $\left\{u^{n_{1}}\right\}$ converges uniformly on compact sets to an $\alpha$-Hölder continuous function $u$. Moreover $|u|_{\alpha} \leqslant K_{1}$.

By Lemma $5,\left|q_{x}^{n}\right| \leqslant K_{5}(\varepsilon)\left|u_{x}^{n}\right|_{\infty}$, thus $N_{n}=\max \left|q_{x}^{n}\right| \leqslant K_{5}(\varepsilon)\left|u_{x}^{n}\right|_{\infty}$ is also finite. If we put $c=\frac{1}{2} K_{5}^{-2}$ in Lemma 2 , we obtain

$$
\left|u_{x}^{n}\right|^{2} \leqslant K_{2}+2 K_{3} K_{5}^{2}+\left(\frac{1}{2} \frac{1}{K_{5}^{2}}\right)\left(K_{5}^{2}\left|u_{x}^{n}\right|_{k}^{2} \infty\right) \text {. }
$$


Taking the supremum in the left-hand side we have $\left|u_{x}^{n}\right|_{\infty}^{2} \leqslant 2\left(K_{2}+2 K_{3} K_{5}^{2}\right)$ so that $\left|q_{x}^{n}\right|_{\infty}^{2} \leqslant 2 K_{5}\left(K_{2}+2 K_{3} K_{5}^{2}\right)$. Thus $\left|q^{n}\right|_{\alpha}$ and thus $\left|q_{(n)}^{n}\right|_{\alpha}$ can be bounded above by a constant $K_{10}$ depending only on $\varepsilon, M_{1}, T$, and $M_{0}$. If we put $v=\left(u^{n}\right)^{2}$ we have $u^{n} v_{x x}+\left(\beta^{n} q_{(n)}^{n}-\mu^{n}\right) v-v_{t}=\varepsilon\left(\beta^{n} q_{(n)}^{n}-\mu^{n}\right) u^{n}$. Since $\left|u^{n}\right|_{\alpha} \leqslant K_{1}, u^{n} \geqslant \varepsilon$ and $\left|q^{n}\right|_{\alpha} \leqslant$ $K_{11}$, we obtain that $|v|_{2+\alpha}$ is bounded and consequently $\left|u^{n}\right|_{2+\alpha}$ is bounded by a constant $K_{12}(\varepsilon)$. Then for $\alpha^{\prime}<\alpha$, there exists a subsequence $\left\{u^{n_{2}}\right\}$ that converges to $u$ in $C^{2+\alpha^{\prime}}\left(\Omega_{T}\right)$. In particular $\left\{u_{x}^{n_{2}}\right\}$ converges uniformly to $u_{x}$ on compact sets. Also $\left\{q_{\left(n_{2}\right)}^{n_{2}}\right\}$ converges pointwise to $q$ and $q^{n_{2}}\left(\beta^{n_{2}} q_{\left(n_{2}\right)}^{n_{2}}-\mu^{n_{2}}\right)$ converges pointwise to $q(\beta q-\mu)$. Therefore all the integrals converge in (28) and (29) and we have a weak solution $u=u_{\varepsilon}$, $q=q_{\varepsilon}$ of the problem (21), (22), that is to say, $u_{\varepsilon}$ and $q_{\varepsilon}$ satisfy

$$
\begin{aligned}
& \iint_{\Omega_{T}}\left[\frac{1}{2}\left(u_{\varepsilon}\right)_{x} \phi_{x}-u_{\varepsilon} \phi_{t}\right] d x d t \\
& \quad=\iint_{\Omega_{T}}\left[\left(\beta\left(u_{\varepsilon}\right) q_{\varepsilon}-\mu\left(u_{\varepsilon}\right)\right)\left(u_{\varepsilon}-\varepsilon\right)\right] \phi d x d t+\int_{\mathbf{R}}\left(u_{0}(x)+\varepsilon\right) \phi(x, 0) d x
\end{aligned}
$$

and

$$
\begin{aligned}
& \iint_{\Omega_{T}}\left[\frac{1}{2}\left(u_{\varepsilon}\right)_{x} q_{\varepsilon} \phi_{x}-u_{\varepsilon} q_{\varepsilon} \phi_{t}\right] d x d t \\
& \quad=\iint_{\Omega_{T}}\left[\left(\beta\left(u_{\varepsilon}\right)-\alpha-\mu\left(u_{\varepsilon}\right)\right)\left(u_{\varepsilon}-\varepsilon\right) q_{\varepsilon} \phi\right] d x d t+\int_{\mathbf{R}}\left(u_{0}(x)+\varepsilon\right) q_{0}(x) \phi(x, 0) d x
\end{aligned}
$$

for every test function $\phi$ over $\Omega_{T}$.

Since $\alpha \in(0,1)$ is arbitrary instead of $\alpha^{\prime}$ we can write again $\alpha$. Moreover $u_{\varepsilon}$ and $q_{\varepsilon}$ satisfy: $u_{\varepsilon} \in C^{2+\alpha}\left(\Omega_{T}\right),\left|u_{\varepsilon}\right|_{2+\alpha} \leqslant K_{12}(\varepsilon), q_{\varepsilon} \in C^{\alpha}\left(\Omega_{T}\right),\left|q_{\varepsilon}\right|_{\alpha} \leqslant K_{11}(\varepsilon)$. In particular $u_{\varepsilon}$ is a classical solution of (6) or (21).

\section{Convergence of the $u_{\varepsilon}, q_{\varepsilon}$ solutions. The main theorem.}

THEOREM 3. Under the given hypotheses on $\beta, \mu, u_{0}$, and $q_{0}$, there exists a weak solution of problem (1) and (2), that is, there is a continuous function $u,\left(u^{2}\right)_{x} \in L_{\mathrm{loc}}^{2}\left(\Omega_{T}\right)$, and a bounded function $q, q \in L_{\mathrm{loc}}^{2}\left(\Omega_{T}\right)$, that satisfy (3) and (4) for every test function $\phi$ over $\Omega_{T}$.

Proof. Again by the Hölder property, $\left|u_{\varepsilon}\right|_{\alpha} \leqslant K_{1}$, so we can extract a subsequence $\left\{u_{\varepsilon_{k}}\right\}$ that converges uniformly on compact sets to an $\alpha$-Hölder continuous function $u$. Moreover, $|u|_{\alpha} \leqslant K_{1}$. Since $\left\{q_{\varepsilon_{k}}\right\}$ is uniformly bounded by 1 , we can extract a subsequence converging weakly to a function $q$ in $L_{\mathrm{loc}}^{2}\left(\Omega_{T}\right)$. We call these sequences again $\left\{u_{k}\right\}$ and $\left\{q_{k}\right\}$ and will prove that a subsequence $\left\{\left(u_{k_{n}}^{2}\right)_{x}\right\}$ of $\left\{\left(u_{k}^{2}\right)_{x}\right\}$ converges pointwise to $\left(u^{2}\right)_{x}$, so that all the integrals in (30) and (31) will converge as $\varepsilon_{k_{n}} \rightarrow 0$ proving that $u$ and $q$ are solutions of (1) and (2). 
Let $Q_{3}$ be a rectangle of length $\frac{1}{2}$ and width $\frac{1}{16}$ centered at $(x, t)=(0,1), Q_{2}=2 Q_{3}$ and $Q_{1}=2 Q_{2}$. Let $f_{1}(x, t), f_{2}(x, t)$ be bounded in $Q_{1}$ with $\left|f_{1}, f_{2}\right| \leqslant K_{21}$. If $z$ is a continuous weak solution in $Q_{1}$ of

$$
\frac{d}{d x}\left(w z_{x}+f_{1}(x, t)\right)+f_{2}(x, t) z=z_{t}
$$

with $\frac{1}{2} \leqslant w \leqslant \frac{3}{2}$ and $|z|_{L^{2}\left(Q_{2}\right)} \leqslant K_{22}$, then by Aronson-Serrin [13] (Theorems 2 and 3) there exist constants $C_{1}, C_{2}$ depending only on $K_{21}, K_{22}$ such that $|z| \leqslant C_{1}$ and $|z|_{\sigma} \leqslant C_{3}$ in $Q_{3}$, for some $\sigma>0$. We use these facts to prove the next lemma.

Lemma 6 (Regularity). If $0<u_{k}\left(x_{0}, t_{0}\right)=\delta<M$, then

$$
\left|\left(u_{k}^{2}\right)_{x}\left(x_{0}, t_{0}\right)\right| \leqslant C_{4}\left(u_{k}\left(x_{0}, t_{0}\right)\right)^{1 / 4}
$$

and

$$
\left|\left(u_{k}^{2}\right)_{x}\right|_{\sigma}=C_{5}\left(K_{2}, M, M_{1}, t_{0}, D_{0}\right)
$$

in a neighborhood $D_{0}$ of $\left(x_{0}, t_{0}\right)$.

Proof. We define a linear change of variables

$$
\begin{aligned}
x(\xi) & =x_{0}+b \delta^{(2+\alpha) / 2} \alpha \xi, \\
t(s) & =t_{0}+b^{2} \delta^{2 / \alpha}(s-1),
\end{aligned}
$$

where $b=\min \left\{\left(8 K_{1}\right)^{-1 / \alpha}, t_{0}, M_{1}^{-2}\right\}$, that maps $Q_{1}$ onto a neighborhood $D_{0}$ of $\left(x_{0}, t_{0}\right)$.

Let $w(\xi, s)=\delta^{-1} u_{k}(x(\xi), t(s))$ for $(\xi, s) \in Q_{1}$. Then $w$ satisfies: $w w_{\xi \xi}+w_{\xi}^{2}+$ $b^{2} \delta^{2 / \alpha} h\left(x, t, u_{k}\right)\left(w-\varepsilon \delta^{-1}\right)=w_{s}$. We put $\bar{h}=b^{2} \delta^{2 / \alpha} h\left(x, t, u_{k}\right)$ and $z=\left(w^{2}\right)_{\xi}$. Differentiating with respect to $\xi$ we get

$$
z_{s}=\left[w z_{\xi}+2 \bar{h}\left(w^{2}-\varepsilon \delta^{-1} w\right)\right]_{\xi} .
$$

By the Hölder property of $u_{k}$ and by the choice of $b,\left|u_{k}(x(\xi), t(s))-u_{k}\left(x_{0}, t_{0}\right)\right| \leqslant \delta / 4$. Thus dividing by $\delta$, we obtain $|w(\xi, s)-1| \leqslant \frac{1}{4}$, and consequently $\frac{1}{2} \leqslant w \leqslant \frac{3}{2}$.

Since $w$ is a solution of (7) in $Q_{1}$ we also have

$$
\iint_{Q_{1}} \frac{1}{2}\left(w^{2}\right)_{\xi} G_{\xi}-w G_{s} d \xi d s=\iint_{Q_{1}} \bar{h}\left(w-\varepsilon \delta^{-1}\right) G d \xi d s
$$

for every test function $G$ that vanishes on the (total) boundary of $Q_{1}$. Let $g(\xi, s)$ be a $C^{\infty}$ function that takes values between 0 and 1 , that is identically 1 in $Q_{2}$ and with derivatives $g_{\xi}, g_{s}$ bounded by 4 . Then the function $G=w^{2} g^{2}$ is an admissible function in (32). This gives

$$
\iint_{Q_{1}} g^{2}\left(\left(w^{2}\right)_{\xi}\right)^{2} d \xi d s \leqslant C_{3}\left(K_{1}, M_{1}, t_{0}\right) .
$$

In particular,

$$
|z|_{L^{2}\left(Q_{2}\right)}=\iint_{Q_{2}}\left(\left(w^{2}\right)_{\xi}\right)^{2} d \xi d s \leqslant C_{3} .
$$


Thus by the Aronson-Serrin theorems, $|z|=\left|\left(w^{2}\right)_{\xi}\right| \leqslant C_{1}$ and $|z|_{\sigma}=\left|\left(w^{2}\right)_{\xi}\right|_{\sigma} \leqslant C_{2}$ in $Q_{3}$. Hence,

$$
\left|\left(u_{k}^{2}\right)_{x}\left(x_{0}, t_{0}\right)\right|=2 b^{-1} \delta^{(3 \alpha-2) / 2} \alpha\left|\left(w^{2}\right)_{\xi}(0,1)\right| \leqslant \frac{2 C_{1}^{1 / 4}}{b}=C_{4}\left(u_{k}\left(x_{0}, t_{0}\right)\right)^{1 / 4}
$$

for $\alpha \geqslant 4 / 5$. Also we can assume that $\sigma$ is smaller than $(3 \alpha-2) / 2 \alpha$. Then

$$
\begin{aligned}
\frac{\left|\left(u_{k}^{2}\right)_{x}\left(x_{1}, t_{1}\right)-\left(u_{k}^{2}\right)_{x}\left(x_{2}, t_{2}\right)\right|}{\left|x_{1}-x_{2}\right|^{\sigma}+\left|t_{1}-t_{2}\right|^{\sigma / 2}} & \leqslant \frac{2 b^{-1} \delta^{(3 \alpha-2) / 2} \alpha\left|\left(w^{2}\right)_{\xi}\left(\xi_{1}, s_{1}\right)-\left(w^{2}\right)_{\xi}\left(\xi_{1}, s_{2}\right)\right|}{b^{\sigma} \delta^{\sigma(2+\alpha) / 2 \alpha}\left(\left|\xi_{1}-\xi_{2}\right|^{\sigma}+\left|s_{1}-s_{2}\right|^{2 / 2}\right)} \\
& \leqslant 2 b^{-1-\sigma} \delta^{[(3 \alpha-2)-\sigma(2+\alpha)] / 2 \alpha} C_{2} \leqslant 2 b^{-1-\sigma} C_{2} M^{2} \\
& =C_{5}\left(K_{2}, M_{1}, M, t_{0}, D_{0}\right) .
\end{aligned}
$$

In particular, $C_{4}$ and $C_{5}$ do not depend on $\delta$.

Differentiability of $u^{2}$. Now we will prove that $u^{2}$ is differentiable with respect to $x$, for every $t>0$. We divide the proof in two cases:

(i) If $u\left(x_{0}, t_{0}\right)=0$, since $u$ is $\alpha$-Hölder continuous in $x,|u(x, t)| \leqslant K_{1}\left|x-x_{0}\right|^{\alpha}$ and

$$
\left|\frac{u^{2}\left(x_{0}, t_{0}\right)-u^{2}\left(x_{0}, t_{0}\right)}{h}\right| \leqslant K_{1} h^{2 \alpha-1} \rightarrow 0
$$

as $h \rightarrow 0$ for $\alpha>1 / 2$.

(ii) If $u\left(x_{0}, t_{0}\right)=\delta>0$, by the uniform continuity of $u$ and the uniform convergence of $\left\{u_{k}\right\}$, there is an integer $k_{0}$ and a neighborhood $D_{0}$ of $\left(x_{0}, t_{0}\right)$ in which

$$
\frac{3}{4} \delta \leqslant u_{k}(x, t) \leqslant \frac{5}{4} \delta \text { for } k \geqslant k_{0} .
$$

By the previous lemma, $\left|\left(u_{k}\right)_{x}\right|_{\sigma} \leqslant C_{5}$ in a subdomain $D_{01}$. Thus we can extract a subsequence $\left\{u_{k_{n}}\right\}$ such that $\left\{\left(u_{k_{n}}^{2}\right)_{x}\right\}$ converges uniformly in a compact subdomain of $D_{01}$ containing $\left(x_{0}, t_{0}\right)$.

Since $\left\{u_{k_{n}}^{2}\right\}$ converges uniformly to $u^{2}$ we conclude that $\left(u^{2}\right)_{x}=\lim _{k_{n} \rightarrow \infty}\left(u_{k_{n}}^{2}\right)_{x}$, for every $x$ in that subdomain. Therefore $\left(u^{2}\right)_{x}$ exists everywhere.

Completion of the proof. Now we fix $R$ and $t_{1}$. We prove that there exists a subsequence $\left\{u_{k, n}\right\}$ of $\left\{u_{k}\right\}$ such that $\left\{\left(u_{k, n}^{2}\right)_{x}\right\}$ converges pointwise to $\left(u^{2}\right)_{x}$ in $D_{R, t_{1}}$.

Let $n \geqslant 2$ and $B_{n}=\left\{(x, t) \in D_{R, t_{1}} / u(x, t) \geqslant 1 / n\right\}$. For $\left(x_{0}, t_{0}\right) \in B_{n}$, with $u\left(x_{0}, t_{0}\right)$ $=\delta \geqslant 1 / n$ as before, there is an integer $k_{0}$ and a neighborhood $D_{0}$, in which $\frac{3}{4} \delta \leqslant$ $u_{k}(x, t) \leqslant \frac{5}{4} \delta$ for $k \geqslant k_{0}$. Then by the regularity lemma there is a $D_{01} \leqslant D_{0}$ such that $\left|\left(u_{k}^{2}\right)_{x}\right|_{\sigma} \leqslant C_{5}$ in $D_{01}$. By compactity we can cover $B_{n}$ with finitely many of these neighborhoods and take $C_{6}$ to be the maximum of the $C_{5}$ 's. Then $\left|\left(u_{k}^{2}\right)_{x}\right|_{\sigma} \leqslant C_{6}$ in $B_{n}$, so there exists a subsequence $\left\{u_{k, n}\right\}$ such that $\left\{\left(u_{k, n}^{2}\right)_{x}\right\}$ converges uniformly in $B_{n}$. Then, out of this sequence and by the same arguments we extract a subsequence $\left\{u_{k, n+1}\right\}$ such that $\left\{\left(u_{k, n+1}^{2}\right)_{x}\right\}$ converges uniformly in $B_{n+1}$. We repeat for every $n$ and pick a diagonal subsequence $\left\{u_{k, k}\right\}$. We claim that $\left\{\left(u_{k, k}^{2}\right)_{x}\right\}$ converges pointwise to $\left(u^{2}\right)_{x}$ in $D_{R, t_{1}}$. Let $\eta>0$ be given. If $u\left(x_{0}, t_{0}\right)=0$, by the differentiability of $u^{2},\left(u^{2}\right)_{x}\left(x_{0}, t_{0}\right)=0$. Since $\left\{u_{k, k}^{2}\right\}$ converges uniformly to $u^{2}$ and $u_{k, k}\left(x_{0}, t_{0}\right)=\delta_{k} \geqslant \varepsilon_{k}>0$, by the regularity lemma 6 and since $\delta_{k} \rightarrow 0,\left|\left(u_{k, k}^{2}\right)_{x}\left(x_{0}, t_{0}\right)\right| \leqslant C_{4} \delta_{k}^{1 / 4} \leqslant \eta$ for $k$ large. If $u\left(x_{0}, t_{0}\right)>0$ we pick a positive integer $r$ such that $u\left(x_{0}, t_{0}\right) \geqslant 1 / r$. Then $\left(x_{0}, t_{0}\right) \in B_{r}$ and since $\left\{u_{k, k}\right\}$ is a 
subsequence of $\left\{u_{k, r}\right\}$ for $k>r$, and $\left\{\left(u_{k, r}^{2}\right)_{x}\right\}$ converges uniformly in $B_{r}$ to $\left(u^{2}\right)_{x}$, we have $\left|\left(u_{k, r}^{2}\right)_{x}\left(x_{0}, t_{0}\right)-\left(u^{2}\right)_{x}\left(x_{0}, t_{0}\right)\right| \leqslant \eta$ for $k$ large, and thus $\mid\left(u_{k, k}^{2}\right)_{x}\left(x_{0}, t_{0}\right)-$ $\left(u^{2}\right)_{x}\left(x_{0}, t_{0}\right) \mid \leqslant \eta$ for $k$ large.

We now finish the proof of the main theorem. We let $t_{1}$ go to 0 and $R$ go to $\infty$ and pick a diagonal subsequence, that we call again $\left\{u_{k}\right\}$. Then the corresponding $\left\{q_{k}\right\}$ converges weakly to $q,\left\{\left(u_{k}^{2}\right)_{x}\right\}$ converges pointwise to $\left(u^{2}\right)_{x},\left\{u_{k}\right\}$ converges uniformly on compact sets to $u$, and $\varepsilon_{k}\left(B\left(u_{k}\right) q_{k}-\mu\left(u_{k}\right)\right)$ converges to 0 . In this form all the integrals converge in (30), (31) and we obtain a weak solution of problem (1) and (2).

The solution we have obtained has the following property: If $u_{0}(x)>0$ in $\left(x_{1}, x_{2}\right)$ and $u_{0}(x)=0$ otherwise, then the set $P[u]=\{(x, t) / u(x, t)>0\}$ is bounded by a decreasing curve $x=\xi_{1}(t)$ through $\left(x_{1}, 0\right)$ and an increasing curve $x=\xi_{2}(t)$ through $\left(x_{2}, 0\right)$. We prove this assertion by means of the next two lemmas.

Lemma 7. Let $t_{0} \in[0, T)$. If $u\left(x_{0}, t_{0}\right)=\eta>0$, then there is a positive constant $K$, independent of $\varepsilon$, such that $u_{\varepsilon}\left(x_{0}, t\right) \geqslant K \eta^{2}$ for every $t \geqslant t_{0}$. In particular, if $u_{0}\left(x_{0}\right)>0$ then $u\left(x_{0}, t\right)>0$ for every $t \geqslant 0$.

Proof. Let $L_{1}[z]=\left(z z_{x}\right)_{x}-N z-z_{t}, N \geqslant 2 M_{1}$, and assume we have a bounded solution $v$ in $\Omega_{T}$ of

$$
\left(v v_{x}\right)_{x}-N v=v_{t}, v(x, 0)=u_{0}(x)+\varepsilon .
$$

Then $L_{1}\left[u_{\varepsilon}\right] \leqslant 0=L_{1}[v]$ and $v(x, 0) \leqslant u_{\varepsilon}(x, 0)$, so by the maximum principle, $v(x, t) \leqslant$ $u_{\varepsilon}(x, t)$ in $\Omega_{T}$. We can obtain solutions of (32) by considering the transformation

$$
w(x, \tau)=\frac{1}{1-N \tau} v(x, t(\tau)), t(\tau)=-\frac{1}{N} \log (1-N \tau) .
$$

Then (32) transforms into

$$
w_{\tau}=\left(w w_{x}\right)_{x}, \quad w(x, 0)=u_{0}(x)+\varepsilon .
$$

Let $w$ be the unique solution of (33) (that exists by Theorem 2 in [12]). This solution is obtained as the limit of solutions to the boundary value problems:

$$
\left(w^{n} w_{x}^{n}\right)_{x}=w_{\tau}^{n}, \quad w^{n}(x, 0)=u_{0}(x)+\varepsilon, \quad w^{n}( \pm n, t)=u_{0}( \pm n)+\varepsilon .
$$

Let $a=x_{0}-\eta / 2 M_{0}, b=x_{0}+\eta / 2 M_{0}$. Since $u_{0}$ is Lipshitz with constant $M_{0}, u_{0}(x) \geqslant$ $\eta / 2$ in $[a, b]$. We consider

$$
\bar{w}=\frac{-2 \eta}{(b-a)^{2}+4 \eta \tau}(x-a)(x-b) \quad \text { and } \quad L_{2}[z]=\left(z z_{x}\right)-z \tau \text {. }
$$

Then $L_{2}\left[w^{\eta}\right]=0 \leqslant L_{2}[\bar{w}], \bar{w}(x, 0) \leqslant \eta / 2 \leqslant w^{\eta}(x, 0)$ and $\bar{w}(a, \tau)=0 \leqslant w^{\eta}(a, \tau)$. Further, $\bar{w}(b, \tau)=0 \leqslant w^{\eta}(b, \tau)$, so $w \leqslant w^{\eta}$ in $[a, b] \times[0, \tau)$ for any $\tau<1 / N$.

At $x=x_{0}$ we obtain

$$
\bar{w}\left(x_{0}, \tau\right)=\frac{\eta(b-a)^{2}}{2(b-a)^{2}+8 \eta \tau} \leqslant w^{\eta}\left(x_{0}, \tau\right) .
$$

Since this holds for every $n$, the same is true for $w$. Thus

$$
u_{\varepsilon}\left(x_{0}, t\right) \geqslant \frac{\eta(b-a)^{2}(1-N \tau)}{2(b-a)^{2}+8 \eta \tau} \geqslant \frac{\eta^{2}}{2} \cdot \frac{N e^{-N T}}{N+2 M_{0}^{2}},
$$

and the result follows with $K=N e^{-N T} / 2\left(N+2 M_{0}^{2}\right)$. 
Lemma 8. For $t_{0} \in[0, T)$, the support of $u\left(x, t_{0}\right)$ is an interval $\left[\xi_{1}\left(t_{0}\right), \xi_{2}\left(t_{0}\right)\right]$.

Proof. Suppose not. Then there exists a point $\left(x_{0}, t_{0}\right)$ for which $u\left(x_{0}, t_{0}\right)=0$ and $u(x, t)$ is positive for some values of $x$ before $x_{0}$ and after $x_{0}$. Pick a point $a>x_{0}$ with $u\left(a, t_{0}\right)=\eta>0$. By Lemma $1, x_{0}>x_{2}$ and $u\left(x_{0}, t\right)=0$ for $t \leqslant t_{0}$. By the continuity of $u$ and the uniform convergence of $\left\{u_{\varepsilon_{k}}\right\}$ for $k$ larger than a certain $k_{0}, u_{\varepsilon_{k}}\left(x_{0}, t\right) \leqslant \eta / 4$ and there is a neighborhood $D_{0}$ containing $\left(a, t_{0}\right)$ in which $u_{\varepsilon_{k}}(x, t)>\eta / 2$. We also pick $k_{0}$ large such that $\varepsilon_{k} \leqslant \eta / 4$. Then in the domain $\left[x_{0}, \infty\right) \times\left[0, t_{0}\right), u_{\varepsilon_{k}} \leqslant \eta / 4$ on the lower and left boundaries, and $u_{\varepsilon_{k}}$ tends to $\varepsilon_{k} \leqslant \eta / 4$ as $|x| \rightarrow \infty$, so by the maximum principle, $u$ cannot be larger than $\eta / 2$. This contradiction proves the lemma.

\section{REFERENCES}

[1] M. E. (jurtin. Some questions and open problems in continuum mechanics and population dinamics, J. Diff. Eq. 48. 293-312 (1983)

[2] M. E. Gurtin, A sistem of equations for age dependent population diffusion, J. Theor. Biol. 40, 389-392 (1973)

[3] M. E. Gurtin and R. C. MacCamy, Nonlinear age dependent population dinamics, Arch. Rat. Mech. Anal. 54. $281-300(1974)$

[4] M. E. Gurtin and R. C. MacCamy, On the diffusion of biological populations, Math. Bioscience 33, 35-49 (1977)

[5] M. E. Gurtin and R. C. MacCamy, Population dinamics with age dependence, Nonlinear Analysis and Mech. Heriot-Watt Symposium, Vol. III, Pitman, 1979

[6] M. E. Gurtin and R. C. MacCamy, Some simple models for nonlinear age-dependent population dynamics, Math. Biosciences 43, 199-211 (1979)

[7] M. E. Gurtin and R. C. MacCamy, Diffusion models for age-structured population, Math. Biosciences 54. $49-59(1981)$

[8] R. C. MacCamy, A population model with nonlinear diffusion, J. Diff. Equations 39, 52-72 (1981)

[9] (3. E. Hernandez, Existence of solutions of population dinamics problems with diffusion. Thesis, University of Minnesota, 1983

[10] A. Fricdman, Partial differential equations of parabolic tlpe, Prentice-Hall, 1964

[11] O. A. Ladyženskaja, V. A. Solonikov and N. N. Ural'ceva, Linear and quasilinear equations of parabolic type. Transl. Math. Monographs, vol. 23, Amer. Math. Soc., 1968

[12] D. (;. Aronson, Regularity properties of flow's through porous media: a counterexample, SIAM J. Appl. Math. 19 (1970)

[13]D. (i. Aronson and J. Serrin, Local hehavior of solutions of quasilinear parabolic equations. Arch. Rat. Mech. Anal. 25, 81-122 (1967) 